\title{
Disseminated Mycobacterium szulgai involving lung, lymph nodes and bone: a case report
}

\author{
Lei Zhang", Kai Liu", Lirong Liu, Chong Meng, Yongxing Chen \\ Department of Pulmonary and Critical Care Medicine, Hainan General Hospital, Hainan Affiliated Hospital of Hainan Medical University, Haikou, \\ China \\ \#These authors contributed equally to this work. \\ Correspondence to: Yongxing Chen. Department of Pulmonary and Critical Care Medicine, Hainan General Hospital, Hainan Affiliated Hospital of \\ Hainan Medical University, Haikou 570311, China. Email: yxchenhx@163.com.
}

\begin{abstract}
Nontuberculous mycobacteria (NTM) refer to a large group of mycobacteria other than Mycobacterium tuberculosis complex and Mycobacterium leprae. Mycobacterium szulgai (M. szulgai) is a slow growing species of nontuberculous mycobacteria (NTM), which can cause infection in multiple organs, including the lungs. Using the technique of next-generation sequencing (NGS), we diagnosed disseminated M. szulgai infection in a patient with no obvious immunodeficiency. We report on a 66-year-old female patient who presented with enlarged cervical lymph nodes and an intermittent fever. Imaging showed multiple, enlarged, abnormal lymph nodes, a pulmonary mass and rib lesions that strongly suggested neoplasia. There was no significant improvement in symptoms after intermittent antibiotic treatment. The pathological results of multiple biopsies did not support the diagnosis of tumors. The diagnosis of M. szulgai infection was confirmed by NGS. The patient started standard treatment with clarithromycin, ethambutol, and moxifloxacin in July 2020. Since then and over the 10-month follow-up period, there has been a progressive reduction in the size of the enlarged lymph nodes and lung lesions, and no recurrence of fever or other symptoms. M. szulgai is a potential cause of infection (including of disseminated disease) even in patients with no obvious immunosuppression. The potential usefulness of the NGS of clinical samples should be highlighted.
\end{abstract}

Keywords: Nontuberculous mycobacteria (NTM); Mycobacterium szulgai (M. szulgai); next-generation sequencing (NGS); case report

Submitted Nov 18, 2021. Accepted for publication Jan 18, 2022.

doi: $10.21037 /$ atm-22-105

View this article at: https://dx.doi.org/10.21037/atm-22-105

\section{Introduction}

Nontuberculous mycobacteria (NTM) refer to a large group of mycobacteria other than Mycobacterium tuberculosis complex and Mycobacterium leprae. NTM can be divided into slow growing, and fast-growing species (1-3). Mycobacterium szulgai (M. szulgai) is a slow growing species of NTM $(4,5)$. In a report of $>36,000$ NTM samples from 14 countries in Europe and the Middle East, M. szulgai accounted for $<0.2 \%$ of the isolated strains $(4,5)$. It not only causes lung infections but can also infect other organs (4-7). The clinical symptoms include one or more of the following manifestations, such as fever, cough, sputum expectoration, shortness of breath, skin ulcers, abscesses, etc., which are not specific (4-7). Since the clinical manifestations of nontuberculous mycobacterial infection are similar to those of tuberculosis infection, which is hard to be clinically distinguished, and most of non-tuberculous mycobacterium infection also has positive result of sputum tuberculosis smear, therefore, finding the pathogenic bacteria has become the key to diagnosis and treatment. We report on an unusual case of a patient with no obvious immunodeficiency, who was found to have multiple organ infections caused 


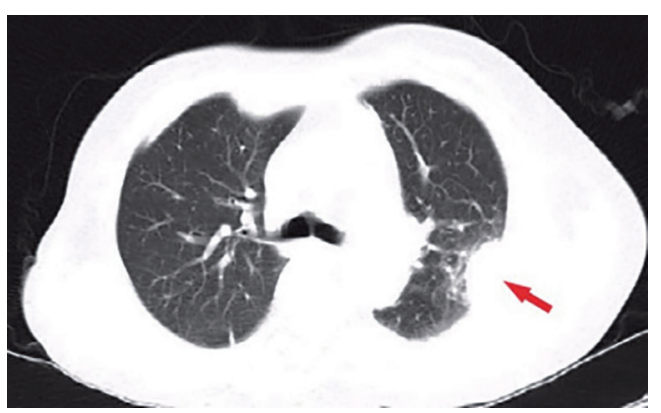

Figure 1 A CT scan of the patient's lungs in May 2020. Red arrow showed a $1.4 \mathrm{~cm} \times 3.6 \mathrm{~cm}$ lesion of soft tissue density in the apico-posterior segment of the left upper lobe. CT, computed tomography.

by $M$. szulgai through next generation sequence (NGS) technology. We present the following article in accordance with the CARE reporting checklist (available at https://atm. amegroups.com/article/view/10.21037/atm-22-105/rc).

\section{Case presentation}

All procedures performed in this study were in accordance with the ethical standards of the institutional and/or national research committee(s) and with the Helsinki Declaration (as revised in 2013). Written informed consent was obtained from the patient for publication of this case report and accompanying images. A copy of the written consent is available for review by the editorial office of this journal.

A 66-year-old female patient was admitted to our hospital with enlarged cervical lymph nodes and an intermittent fever for over 3 months. The lymph nodes in her neck, which were more marked on the left side, had been found to be enlarged and tender some 3 months earlier. Initially, no medication was administered. Despite analgesia, the pain in the lymph nodes in the right side of the neck and left axilla gradually got worse, and was accompanied by left chest pain and intermittent fever. A maximum body temperature of $38.9^{\circ} \mathrm{C}$ was recorded. Computed tomography (CT) scans of the lungs in May 2020 showed a $1.4 \mathrm{~cm} \times 3.6 \mathrm{~cm}$ lesion of soft tissue density in the apico-posterior segment of the left upper lobe (Figure 1). After the administration of piperacillin $(2.5 \mathrm{~g} \mathrm{q} 8 \mathrm{~h})$ and moxifloxacin $(0.4 \mathrm{~g} \mathrm{qd})$ antiinfective treatment for 8 days at a local hospital, the patient's temperature decreased, but an intermittent fever persisted. Fluorodeoxyglucose (FDG) positron emission tomography (PET)-CT, carried out in June 2020 at our hospital, showed an enlargement of the lymph nodes in the right side of the neck, the left axilla, the left side of the mediastinum, and the right inguinal region with increased FDG uptake. The 3rd and 4th ribs on the left were found to be destroyed with adjacent lung tissue involvement. Many parts of the bony skeleton showed patchy uneven densities with increased FDG uptake. These findings suggested a diagnosis of neoplasia (either multiple myeloma or secondary malignant bone deposits). However, a needle aspiration biopsy of a left axillary node and of the left lung only revealed reactive lymph node hyperplasia and scattered mixed inflammatory cell infiltration in the left lung. No clear evidence of malignancy was discovered in either of the specimens. A bone marrow biopsy revealed that the ratio of granulocytes was roughly normal, and no evidence of a malignant tumor, lymphoma, or multiple myeloma was found.

The fever reappeared 6 days before admission to our hospital in June. It occurred mostly in the afternoons and at nights, with a peak temperature of $38.5^{\circ} \mathrm{C}$. The patient had an occasional cough with white sticky sputum. The CT scan was repeated, and showed an enlargement $(7.9 \mathrm{~cm} \times$ $5.6 \mathrm{~cm}$ ) of the original lesion in the apico-posterior segment of the left upper lobe. As neoplastic lesions could not be excluded, the patient was admitted to our hospital in June for diagnosis and further treatment.

The patient had suffered from hypertension and type II diabetes for over 10 years. She had stable blood pressure, and her blood glucose was controlled by diet and exercise alone. She had a history of herpes zoster 1 year beforehand with minor residual neuralgia affecting the left side of the neck. She was a non-smoker and drank no alcohol. No family history of disease was recorded.

The physical examination revealed pale lips and conjunctivae. Several enlarged lymph nodes were palpable on the left side of the neck, fusing into a mass, the largest of which was about $3 \mathrm{~cm} \times 3 \mathrm{~cm}$, hard in nature, and not clearly distinct from the surrounding tissues. An enlarged lymph node, about $2 \mathrm{~cm} \times 2 \mathrm{~cm}$, with tough texture and indistinct borders could also be palpated on the right side of the neck. A hard lymph node, about $3 \mathrm{~cm} \times 2 \mathrm{~cm}$ in size, with distinct borders was also palpable in the left axilla. On the auscultation of both lungs, the breath sounds were coarse with a few moist crackles heard at both lung bases. No positive signs were found in the examinations of the other systems.

The initial laboratory investigations revealed the following results: white blood cell count $18.23 \times 10^{9} / \mathrm{L}$; neutrophil count $11.52 \times 10^{9} / \mathrm{L}$; hemoglobin $72 \mathrm{~g} / \mathrm{L}$; CRP 


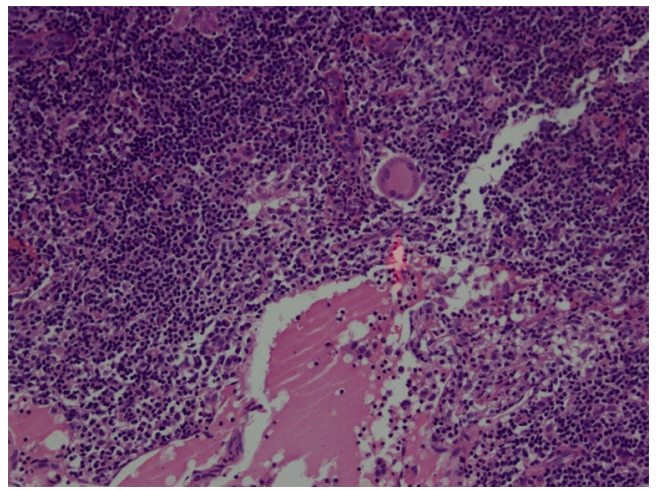

Figure 2 Histopathology of the left neck lymph node showed granulomatous changes (HE staining, $\times 10$ ).

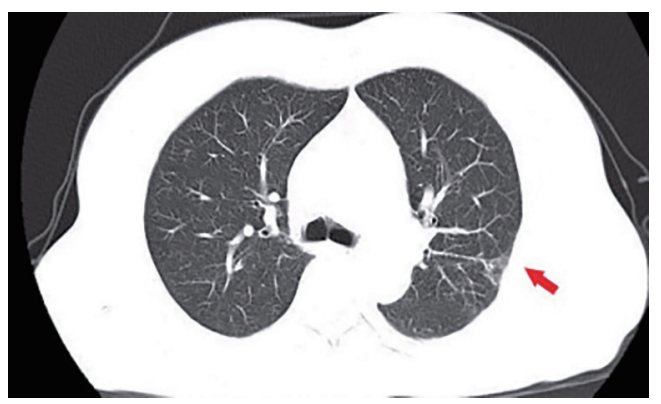

Figure 3 The original lesion in the left lung (red arrow) was improved at 7 months compared to the pre-treatment scan.

$105.91 \mathrm{mg} / \mathrm{L}$; ESR $58 \mathrm{~mm} / \mathrm{h}$; immunoglobulin G $20.96 \mathrm{~g} / \mathrm{L}$ (7.0-16.0 g/L), immunoglobulin A $2.76 \mathrm{~g} / \mathrm{L}(0.7-5.0 \mathrm{~g} / \mathrm{L})$, immunoglobulin $M 1.15 \mathrm{~g} / \mathrm{L}(0.4-2.8 \mathrm{~g} / \mathrm{L})$, light chain $\kappa$ (kappa) $4.97 \mathrm{~g} / \mathrm{L}(2-4.4 \mathrm{~g} / \mathrm{L})$, and light chain $\lambda$ (lambda) $3.56 \mathrm{~g} / \mathrm{L}(1.1-2.4 \mathrm{~g} / \mathrm{L})$. Tuberculin pure protein derivative test (PPD) was strongly positive. The Mantoux was approximately $22 \mathrm{~mm}$ in diameter, and without blistering or lymphangitis. Bence Jones protein was not detected in the urine. The test results for tumor markers, human immunodeficiency virus, a routine sputum smear, and cultures were all negative.

A regular culture of a bronchoalveolar lavage fluid (BALF) specimen obtained at bronchoscopy from the apico-posterior segment of the left upper lobe proved negative 7 days later. A bone marrow biopsy revealed that the ratio of granulocytes was roughly normal. A needle aspiration biopsy of a left axillary node and of the left lung only showed reactive lymph node hyperplasia and scattered mixed inflammatory cell infiltration in the left lung. The enlarged lymph node in the left neck was completely removed surgically and was sent for next-generation sequencing (NGS) and histopathology. NGS refers to a high-throughput technology that determines the nucleic acid sequences extracted from biological samples and yields information on the type and abundance of microorganisms contained in the sample (8). Histopathology results of the left neck lymph node showed granulomatous changes (Figure 2). The immunohistochemistry and special stains were negative. The presence of $M$. szulgai in the lymph node specimen was confirmed 5 days later by NGS.

Standard treatment with clarithromycin (500 mg bid), ethambutol (750 mg qd), and moxifloxacin (0.4 g qd) began in July 2020 according to national and domestic guidelines $(3,9)$. At the beginning of the treatment, the patient experienced some nausea and a poor appetite, which were considered side effects of the drugs. After expectant treatment for 2 weeks, the patient tolerated the medication well. The CT scan was repeated after treatment for 1 month, and then at 3,5, and 7 months, respectively. The lesions in the left lung improved at 1 month and further improved at 7 months compared to the pre-treatment scan (Figure 3). The patient's anemia was resolved; her hemoglobin levels increased to normal and remained stable. The patient's $\mathrm{C}$ reactive protein (CRP) and erythrocyte sedimentation rate (ESR) levels normalized. The pain in the lymph nodes improved significantly, and the patient stopped taking painkillers. There was no recurrence of cough, sputum, or fever. The follow-up period thus far is over 10 months and will continue after the end of treatment.

\section{Discussion}

M. szulgai was named after Dr. Szulga, a Polish microbiologist, who developed the lipid analysis method for identifying this pathogen (10). It is known to be primarily present in environmental sources, such as fresh water, and to affect tropical fish and plants, which have been suggested as potential sources of human infection (11). At present, no human-to-human transmission has been reported (4-6). It is an unusual pathogen that accounts for $<1 \%$ of all human isolates of NTM infection worldwide (12). In China, only 5 provinces have reported isolation of M. szulgai (13).

As techniques of pathogen detection gradually improve, more cases of M. szulgai have been reported, including infections of the lung, skin, urinary tract, osteomyelitis, and tenosynovitis $(4-7,14,15)$. Some of the patients had structural lung disease or reported tobacco use; however, very few cases of immunocompetent patients 
have been reported over the last 4 decades. In the last century, Maloney reported single organ involvement, lung disease, lymphadenitis, and cutaneous involvement, but also recorded rare reports of disseminated disease (16). However, in a recent discussion of the literature, Weng reported that osteomyelitis, cutaneous involvement, and lymphadenopathy were the most commonly involved sites in populations with disseminated M. szulgai (17). M. szulgai rarely causes pulmonary infection, and less commonly causes extrapulmonary disease, and even more rarely causes systemic infections.

In our case, a 66-year-old female patient, without cancer or immunodeficiency, apart from controlled type II diabetes, presented suffering from a recurrent fever, cough, and sputum expectoration, with anemia, and also pain in multiple lymph nodes. FDG PET-CT scans showed multiple high-uptake lesions and bone destruction, while CT scans indicated a non-specific lung lesion and lymphadenopathy. This patient's diagnosis of M. szulgai infection was based on the clinical presentation, imaging evidence, a consistent lymph node histology, which indicated granulomatous changes, and NGS. The marked clinical response and continuing improvement in imaging following treatment also strongly support the diagnosis. Diabetes was considered a possible predisposing factor as the patient had a history of herpes zoster.

The presentation of a fever, cough, and sputum did not differ from common pneumonia. Thus, the determination of the specific etiology was crucial. The clinical practice expert consensus for the application of metagenomic NGS (18), recommends its use as the first choice of second line investigations for patients suspected of focal infection, after routine biochemistry, cultures, or polymerase chain reaction (PCR) inspections of the focal area, if they do not yield an etiological diagnosis. In this case, the differential diagnoses that needed to be considered included systemic metastases of a malignant tumor, lymphoma, and multiple myeloma. We carried out multiple pathological examinations of multiple tissues, including bone marrow, lymph nodes, and lung biopsies, to confirm or exclude the diagnosis of neoplasia. No evidence of malignancy was found, and an infectious etiology was considered. Eventually, NGS was carried out, and M. szulgai infection was diagnosed.

The clinical manifestations and histopathology of NTM, especially M. szulgai, infection lack specificity. The diagnosis mainly relies on a pathologic examination and mycobacterial culture. The BALF specimen of our patient eventually showed a negative mycobacterial culture. The traditional method for identifying NTM relies on the characteristics of bacterial growth and biochemical reaction, which usually takes several weeks. The identification results may be affected by many factors. In recent years, molecular biology methods have gradually been adopted for the identification of nontuberculous mycobacteria $(3,7,19)$. For patients with respiratory infections, if a clear etiological basis has not been obtained through traditional laboratory examinations within 3 days and empirical antiinfective treatment is ineffective, it is recommended to obtain respiratory tract specimens for NGS (A, II) (18). According to national and domestic guidelines, as NTM is usually resistant to common anti-tuberculosis drugs, once NTM disease is diagnosed, routine anti-tuberculosis drug sensitivity testing is not necessary. In similar cases, triple or quadruple anti-tuberculosis treatments have been chosen $(15,20)$. However, clarithromycin and azithromycin are macrolide drugs, which have been of vital importance in the treatment of NTM over the past 20 years. A total treatment period of $>12$ months after the cultures become negative is suggested (3). Others have shown that treatment, which includes clarithromycin, has achieved good results (17). In this patient, triple therapy was instituted, leading to a significant improvement in fever, pain, anemia, and lesion absorption. The patient's hemoglobin rose from 72 to $145 \mathrm{~g} / \mathrm{L}$. The inflammation indicators also normalized. Continuous follow-up will be arranged.

M. szulgai disseminated infections have been reported worldwide, but cases have rarely been recorded in China, especially in patients without severe immunodeficiency. In this case, the involvement of multiple organs almost led to a misdiagnosis of a tumor. In patients who suffer from recurrent fever with multiple pathological lesions and multiple organ involvement, in addition to repeated investigations, tissue biopsies should be performed and consideration given to bacterial and fungal cultures.

\section{Conclusions}

In conclusion, NTM, including $M$. szulgai, are very uncommon potential causes of infection, even in patients without severe immunosuppression. This report highlights that continued clinical awareness is required to identify rare cases caused by M. szulgai, and the potential usefulness of the NGS of clinical samples in this context.

\section{Acknowledgments}

Funding: None. 


\section{Footnote}

Reporting Checklist: The authors have completed the CARE reporting checklist. Available at https://atm.amegroups. com/article/view/10.21037/atm-22-105/rc

Conflicts of Interest: All authors have completed the ICMJE uniform disclosure form (available at https://atm. amegroups.com/article/view/10.21037/atm-22-105/coif). The authors have no conflicts of interest to declare.

Ethical Statement: The authors are accountable for all aspects of the work in ensuring that questions related to the accuracy or integrity of any part of the work are appropriately investigated and resolved. All procedures performed in this study were in accordance with the ethical standards of the institutional and/or national research committee(s) and with the Helsinki Declaration (as revised in 2013). Written informed consent was obtained from the patient for publication of this case report and accompanying images. A copy of the written consent is available for review by the editorial office of this journal.

Open Access Statement: This is an Open Access article distributed in accordance with the Creative Commons Attribution-NonCommercial-NoDerivs 4.0 International License (CC BY-NC-ND 4.0), which permits the noncommercial replication and distribution of the article with the strict proviso that no changes or edits are made and the original work is properly cited (including links to both the formal publication through the relevant DOI and the license). See: https://creativecommons.org/licenses/by-nc-nd/4.0/.

\section{References}

1. Bennett JE, Dolin R, Blaser MJ. Mandell, douglas, and bennett's principles and practice of infectious diseases. 9th ed. Philadelphia: Elsevier Health Sciences, 2019.

2. Griffith DE, Aksamit T, Brown-Elliott BA, et al. An official ATS/IDSA statement: diagnosis, treatment, and prevention of nontuberculous mycobacterial diseases. Am J Respir Crit Care Med 2007;175:367-416.

3. Chinese Society for Tuberculosis of Chinese Medical Association. Interpretation of expert consensus on the diagnosis and treatment of nontuberculous mycobacterial diseases. Chinese Journal of Tuberculosis and Respiratory Diseases 2012;35:572-80.

4. Manalac TC, Bonilla H. Disseminated Mycobacterium szulgai infection: case report and review of literature. Infect Dis Clin Pract 2007;15:341-4.

5. Khan S, Clerk N, Galloway K, et al. Pulmonary Mycobacterium szulgai infection: a case report. QJM 2016;109:489-90.

6. Gutierrez M, Feola M, Lenge L, et al. First pulmonary case reported in Argentina of infection with mycobacterium szulgai, a rare pathogen. J Clin Microbiol 2007;45:3121-4.

7. Wang $M$, Liu $\mathrm{H}$, Wang $\mathrm{X}$, et al. The next-generation sequencing technology for diagnosis of pulmonary tuberculosis and pulmonary nontuberculous mycobacterial diseases: two cases report and literature review. Anhui Medical Journal 2020;41:610-1.

8. Alekseyev YO, Fazeli R, Yang S, et al. A Next-Generation Sequencing Primer-How Does It Work and What Can It Do? Acad Pathol 2018;5:2374289518766521.

9. Daley CL, Iaccarino JM, Lange C, et al. Treatment of Nontuberculous Mycobacterial Pulmonary Disease: An Official ATS/ERS/ESCMID/IDSA Clinical Practice Guideline. Clin Infect Dis 2020;71:e1-36.

10. Marks J, Jenkins PA, Tsukamura M. Mycobacterium szulgai--a new pathogen. Tubercle 1972;53:210-4.

11. Abalain-Colloc ML, Guillerm D, Saläun M, et al. Mycobacterium szulgai isolated from a patient, a tropical fish and aquarium water. Eur J Clin Microbiol Infect Dis 2003;22:768-9.

12. McSweeney FG, O'Brien ME, Sheehan S, et al. Pulmonary Mycobacterium szulgai infection. Ir Med J 2012;105:275-7.

13. Xiao S, Zhao X, Liu Z, et al. Antibiotic susceptibility of reference and clinical strains of slowly growing nontuberculous mycobacteria of 5 species rarely isolated in China. Disease Surveillance 2020;35:435-41.

14. Oztug Onal C, Kibaroglu E, Kibaroglu E. Nontuberculous mycobacterial pulmonary disease secondary to Mycobacterium szulgai. J Clin Case Rep 2014;4:2.

15. Shamriz O, Engelhard D, Rajs AP, et al. Mycobacterium szulgai chronic multifocal osteomyelitis in an adolescent with inherited STAT1 deficiency. Pediatr Infect Dis J 2013;32:1345-7.

16. Maloney JM, Gregg CR, Stephens DS, et al. Infections caused by Mycobacterium szulgai in humans. Rev Infect Dis 1987;9:1120-6.

17. Weng TP, Syue LS, Lee NY. Disseminated Mycobacterium szulgai infection in a patient with anti-interferon-gamma autoantibodies. IDCases 2020;21:e00848. 
18. Editorial Board of Chinese Journal of Infectious Diseases. Expert consensus on the clinical application of Chinese metagenomics second-generation sequencing technology to detect infectious pathogens. Chinese Journal of Infectious Diseases 2020;38:681-9.

19. Tang S. Interpretation of expert consensus on the diagnosis and treatment of nontuberculous mycobacterial diseases.

Cite this article as: Zhang L, Liu K, Liu L, Meng C, Chen Y. Disseminated Mycobacterium szulgai involving lung, lymph nodes and bone: a case report. Ann Transl Med 2022;10(3):155. doi: 10.21037/atm-22-105
Chinses Journal of Medicine 2016;51:21-4.

20. Meyer JJ, Gelman SS. Multifocal osteomyelitis due to Mycobacterium szulgai in a patient with chronic lymphocytic leukemia. J Infect 2008;56:151-4.

(English Language Editor: L. Huleatt) 